\title{
X-ray mirror development and production for the Athena telescope
}

\section{Maximilien Collon, Ljubiša Babic, Nicolas Barrière, Alex Bayerle, Luigi Castiglione, et al.}

Maximilien J. Collon, Ljubiša Babic, Nicolas M. Barrière, Alex Bayerle, Luigi Castiglione, Noë Eenkhoorn, David Girou, Ramses Günther, Enrico Hauser, Yvette Jenkins, Boris Landgraf, Laurens Keek, Ben Okma, Gregorio Mendoza Serano, Aniket Thete, Giuseppe Vacanti, Sjoerd Verhoeckx, Mark Vervest, Luc Voruz, Marco Beijersbergen, Marcos Bavdaz, Eric Wille, Ivo Ferreira, Sebastiaan Fransen, Brian Shortt, Mark Olde Riekerink, Jeroen Haneveld, Arenda Koelewijn, Maurice Wijnperle, Jan-Joost Lankwarden, Bart Schurink, Ronald Start, Coen van Baren, Paul Hieltjes, Jan Willem den Herder, Evelyn Handick, Michael Krumrey, Miranda Bradshaw, Vadim Burwitz, Sonny Massahi, Sara Buur Svendsen, Desiree Della Monica Ferreira, Finn E. Christensen, Giuseppe Valsecchi, Geeta Kailla, William Mundon, Gavin Phillips, Ian Chequer, Kevin Ball, "X-ray mirror development and production for the Athena telescope," Proc. SPIE 11852, International Conference on Space Optics - ICSO 2020, $118521 Z$ (11 June 2021); doi: 10.1117/12.2599339 


\section{International Conference on Space Optics-ICSO 2020}

Virtual Conference

30 March-2 April 2021

Edited by Bruno Cugny, Zoran Sodnik, and Nikos Karafolas
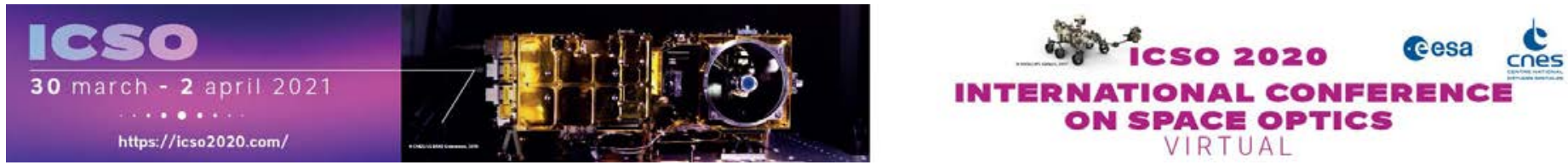

\section{$X$-ray mirror development and production for the Athena telescope}

\section{Cesa isso procedings denes}




\title{
X-ray mirror development and production for the Athena telescope
}

\author{
Maximilien J. Collon*1, Ljubiša Babić ${ }^{1}$, Nicolas M. Barrière ${ }^{1}$, Alex Bayerle ${ }^{1}$, Luigi Castiglione ${ }^{1}$, \\ Noë Eenkhoorn ${ }^{1}$, David Girou ${ }^{1}$, Ramses Günther ${ }^{1}$, Enrico Hauser ${ }^{1}$, Yvette Jenkins ${ }^{1}$, Boris Landgraf ${ }^{1}$, \\ Laurens Keek ${ }^{1}$, Ben Okma ${ }^{1}$, Gregorio Mendoza Serano ${ }^{1}$, Aniket Thete ${ }^{1}$, Giuseppe Vacanti ${ }^{1}$, \\ Sjoerd Verhoeckx ${ }^{1}$, Mark Vervest ${ }^{1}$, Luc Voruz ${ }^{1}$, Marco W. Beijersbergen ${ }^{1}$, \\ Marcos Bavdaz ${ }^{2}$, Eric Wille ${ }^{2}$, Ivo Ferreira ${ }^{2}$, Sebastiaan Fransen ${ }^{2}$, Brian Shortt ${ }^{2}$, \\ Mark Olde Riekerink ${ }^{3}$, Jeroen Haneveld ${ }^{3}$, Arenda Koelewijn ${ }^{3}$, Maurice Wijnperle ${ }^{3}$, \\ Jan-Joost Lankwarden ${ }^{3}$, Bart Schurink ${ }^{3}$, Ronald Start ${ }^{3}$, \\ Coen van Baren ${ }^{4}$, Paul Hieltjes ${ }^{4}$, Jan Willem den Herder ${ }^{4}, 10$, \\ Evelyn Handick ${ }^{5}$, Michael Krumrey ${ }^{5}$, \\ Miranda Bradshaw ${ }^{6}$, Vadim Burwitz ${ }^{6}$, \\ Sonny Massahi ${ }^{7,11}$, Sara Svendsen ${ }^{7}$, Desirée Della Monica Ferreira ${ }^{7}$, Finn E. Christensen ${ }^{7,11}$, \\ Giuseppe Valsecchi ${ }^{8}$, \\ Geeta Kailla ${ }^{9}$, William Mundon ${ }^{9}$, Gavin Phillips ${ }^{9}$, Ian Chequer ${ }^{9}$, Kevin Ball ${ }^{9}$ \\ ${ }^{1}$ cosine, Oosteinde 36, 2361 HE Warmond, The Netherlands \\ ${ }^{2}$ European Space Agency, ESTEC, Keplerlaan 1, 2200 AG Noordwijk, The Netherlands \\ ${ }^{3}$ Micronit B.V., Colosseum 15, 7521 PV Enschede, The Netherlands \\ ${ }^{4}$ SRON, Sorbonnelaan 2, 3584 CA Utrecht, The Netherlands \\ ${ }^{5}$ Physikalisch-Technische Bundesanstalt (PTB), Abbestr. 2-12, 10587 Berlin, Germany \\ ${ }^{6}$ MPI f. extraterrestrische Physik, Giessenbachstrasse 1, 85748 Garching, Germany \\ ${ }^{7}$ DTU Space, Technical University of Denmark, Building 327, DK - 2800 Kgs. Lyngby, Denmark \\ ${ }^{8}$ Media Lario S.r.l., Località Pascolo, 23842 Bosisio Parini(LC), Italy \\ ${ }^{9}$ Teledyne imaging Ltd., 106 Waterhouse Lane, Chelmsford, Essex CM1 2QU, England \\ ${ }^{10}$ Anton Pannekoek Institute, University of Amsterdam, 1090 GE Amsterdam, the Netherlands \\ ${ }^{11}$ CHEXS, Diplomvej 373B, 2800 Kgs. Lyngby, Denmark
}

\begin{abstract}
Athena will be the largest space-based x-ray telescope to be flown by the European Space Agency: its large $2.6 \mathrm{~m}$ diameter lens will use a revolutionary new modular technology, Silicon Pore Optics (SPO). The lens will consist of several hundreds of smaller x-ray lenslets, called mirror modules, which each consist of about 70 mirror pairs. Those mirror modules are arranged in circles in a large optics structure and will focus x-ray photons with an energy of 0.5 to 10 $\mathrm{keV}$ at a distance of $12 \mathrm{~m}$ onto the detectors of Athena. The point-spread function (PSF) of the optic shall achieve a halfenergy width (HEW) of 5" at an energy of $1 \mathrm{keV}$, with an effective area of about $1.4 \mathrm{~m}^{2}$, corresponding to several hundred $\mathrm{m}^{2}$ of super-polished mirrors with a roughness of about $0.3 \mathrm{~nm}$ and a thickness of only $150 \mu \mathrm{m}$. SPO using the highest grade double-side polished $300 \mathrm{~mm}$ wafers commercially available, have been invented to enable such telescopes. SPO allows the cost-effective production of high-resolution, large area, x-ray optics, by using all the advantages that mono-crystalline silicon and the mass production processes of the semi-conductor industry provide. SPO has also shown to be a versatile technology that can be further developed for gamma-ray optics, medical applications and for material research.
\end{abstract}

This paper will present the status of the technology and of the mass production capabilities, show latest performance results and discuss the next steps in the development.

Keywords: x-ray optics, x-ray astronomy, silicon, wafer, stack, pore optics, x-ray telescopes, ATHENA, ARCUS, SPO

1*m.collon@cosine.nl 


\section{INTRODUCTION}

The hot and energetic universe, in which stars are created, galaxies are formed containing black holes and where stars explode in supernovae, can be observed when collecting the x-rays emitted by those extremely hot sources. As the x-rays are absorbed by the Earth's atmosphere, $\mathrm{x}$-ray telescopes need to operate in space. Several imaging X-ray telescopes have been flown since the 1960s, of which many use a so-called Wolter-1 configuration for their optics, which uses a doublereflection grazing incidence angle pair of a parabolic and a hyperbolic shaped mirror to image the astronomical source onto the detector.

The quality of the mirror surface in terms of figure accuracy determines the resolution of the telescope and the roughness of the mirror determines the efficiency, with which the x-rays are reflected. As the grazing incidence angle is typically only of the order of half a degree, the effective geometric mirror surface area of each mirror is low. In order to collect photons to observe faint $\mathrm{x}$-ray sources or to perform spectroscopy one requires therefore a large mirror surface with a roughness of less than $0.5 \mathrm{~nm} \mathrm{rms}$, preferably coated in a high-z material to increase the reflectivity of the mirror in an energy band of 0.1 to $12 \mathrm{keV}$. The angular resolution of the optics is typically expressed in arc seconds and has in past telescopes been limited by the mirror production technology. The total effective area depends on the mirror material and thickness and is limited by the launcher to lift the telescope into space.

The science case for a telescope thus depends on the combination of achievable optics resolution and the effective mirror area, and the available mirror technologies (replication, polishing, slumping, foils) also showed a general correlation: The lighter the mirror, the lower its resolution. The two largest x-ray telescopes launched in 1999 and still in operation serve as examples: The NASA telescope Chandra has an effective mirror area of $800 \mathrm{~cm}^{2}$ at an energy of $250 \mathrm{eV}$, using four super-polished glass shells, 16-24 mm thick, and achieving with those a resolution of 0.5 " half-energy width (HEW). The ESA telescope XMM-Newton uses about 180 replicated nickel shells in three identical telescopes, each shell $0.5-1.0 \mathrm{~mm}$ thin, that together yield an effective area of about $1.500 \mathrm{~cm}^{2}$ at an energy of $1 \mathrm{keV}$, with a resolution of about 13 ".

The European Space Agency (ESA) decided in 2014 to continue studying the hot and energetic universe with a next generation telescope called Athena [1], as part of their Cosmic Vision Program. Athena will essentially consist of one large optic and two detectors and will be the largest X-ray observatory ever flown. The Wide-Field Imager [2] performs high-timing resolution imaging of point sources and sky surveys. The X-ray Integral Field Unit (XIFU [3]) is used for very high-resolution spectroscopy. Their combined science cases [4] result in an angular resolution requirement of 5" HEW and a desired effective area of about $14.000 \mathrm{~cm}^{2}$ at $1 \mathrm{keV}$ for the optics. This translates into about $300 \mathrm{~m}^{2}$ of superpolished mirror surface and requires a new mirror technology to break the general correlation stated above, to make a light-weight yet stiff mirror, in order to achieve the desired optical performance. This technology is being developed by ESA [5], cosine and its partners, both in industry and academia and is called Silicon Pore Optics (SPO) [6].

SPO is modular, separating the concentric shells of other technologies into smaller x-ray lenses. Those are called Mirror Modules and are blocks of stacked thin $(0.11$ to $0.17 \mathrm{~mm})$ silicon mirrors interconnected by stiffening ribs. The source material are super-polished $300 \mathrm{~mm}$ wafers from the semi-conductor industry, which have a starting roughness of $0.1 \mathrm{~nm}$ rms and can be procured in large volumes off-the-shelf. The semi-conductor industry also provides many machines and methods to process the round wafers into coated rectangular mirrors, which are then bent and stacked by robots into the desired figure. For Athena a total of about 150.000 such mirrors will have to be produced and turned into 600 mirror modules, an effort that requires a high degree of automation to remain within reasonable cost and time budgets [7]. The 600 mirror modules are then integrated and co-aligned [8] into a large $2.5 \mathrm{~m}$ diameter structure to form the final optic.

Athena is now in phase B [9] and all critical technologies and detectors need to achieve a Technology Readiness Level (TRL) of 5-6 to pass the next gate, the Mission Adoption Review (MAR) and enter phase C when the flight module will be manufactured. The MAR is planned for November 2022 and Athena should launch in 2033. The unprecedented scale of mass production required for this mission is one of the three critical element for the MAR, as one not only needs to demonstrate that (1) the effective area and (2) the resolution targets for the optics can be met, but also (3) that up to two mirror modules per day can be produced with very high yield in a cost effective manner. Demonstrating that all three goals can be met is part of a technology development program of ESA and this paper reports on the status of the SPO mirror module development. Other critical developments, such as the optical bench and assembly, integration and test (AIT) facilities are reported on elsewhere in this conference [10]. 


\section{STATUS OF SILICON PORE OPTICS IN TERMS OF PERFORMANCE}

The simplest metric to assess the status of the optics development is to report on the performance measured in $\mathrm{x}$-ray. We routinely measure [11] SPO test samples at the XPBF 2.0 (X-ray parallel beam facility) $[12,13]$, operated by PTB at the BESSY II synchrotron radiation facility in Berlin. The facility was recently upgraded and the energy has been changed from $1.6 \mathrm{keV}$ to $1.0 \mathrm{kev}$. Individual stacks of SPOs are co-aligned in double-reflection (becoming an x-ray optical unit (XOU)) using direct $\mathrm{x}$-ray illumination with $0.1 \mathrm{~mm}$ wide $\mathrm{x}$-ray beams that probe the entire surface of the optics with high-spatial resolution. Combining tens of thousands of such measurements allows to determine the overall point spread function (PSF) and to determine its HEW. We routinely also have optics measured at the PANTER facility operated in Munich by the Max Planck Institut für extraterrestrische Physik (MPE) to provide a cross-check and to be able to quantify the effective area and off-axis performance [14, 15].

The development currently used representative radii as proxy for the final Athena configuration, with the so-called middle radius $(\sim 750 \mathrm{~mm})$ being the most advanced. The inner $(\sim 250 \mathrm{~mm})$ and outer $(\sim 1500 \mathrm{~mm})$ radii are also being developed in parallel and lessons learned on each radius can be translated efficiently to other radii.

Over the course of 2020 we have been improving the stacking recipe of the robots to address a number of error components identified in earlier stack generations [16]. We had observed systematic deterioration of the performance on the azimuthal outer edges of the stacks, reducing the usable width of an XOU that has good performance and potentially forcing the scientists having to make a choice between large effective area and high resolution. We can now report that the underlying mechanism that let to these errors has been understood. The solution required development of new oversized mirror plate types with sacrificial lateral extensions (SALEX) parts, that are present during the stacking process, but removed afterwards before the XOUs are integrated into mirror modules. We also improved the stacking equipment to allow finer control of the deposition of the plate edges during the stacking. This has in combination let to a significant improvement of the HEW over $100 \%$ width of an XOU (see Figure 1 right), where for a first time a 20 plate middle radius XOU (S/N 101) in double reflection performs better than 10" (9.4").

Also smaller areas of that XOU-0101 show significant improvement compared to earlier generations. This was achieved by improving the adherence of the optics to the optical design, especially its meridional curvature. The target radius of curvature for an XOU is about $1.6 \mathrm{~km}$, resulting in a meridional sag of about $100 \mathrm{~nm}$, which over a mirror length of 40 $\mathrm{mm}$ is complex to control and set. Too little curvature would limit the XOU HEW to about 5.6", the conical approximation to a Wolter-I system, too large curvature quickly deteriorates the performance further. Figure 1 on the left shows that with improved control over meridional curvature we have been able to pass on a $10 \%$ wide area over 10 plates of the same XOU the 4.3 " barrier, again for the first time.
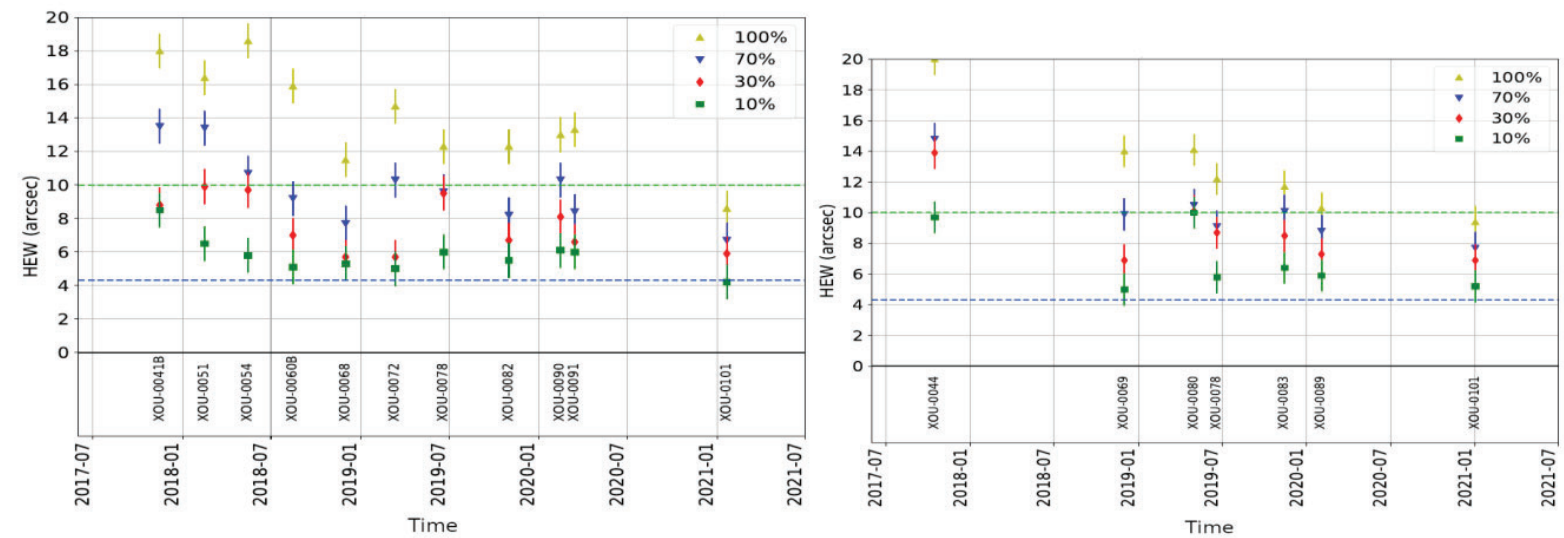

Figure 1: Evolution of the angular resolution (HEW) as a function of time, for 10 plate (left) and 20 plate XOUs (right). Until 2020 all measurements are taken at $1.6 \mathrm{keV}$, as of 2021 we measure at $1.0 \mathrm{keV}$. The measurements report double reflection as measured at BESSY and the four sets of data points report different fractions of contiguous area width over 10 and 20 plate high scan areas. The gap in the middle of 2020 is related to COVID-19 related delays and supply issues. 

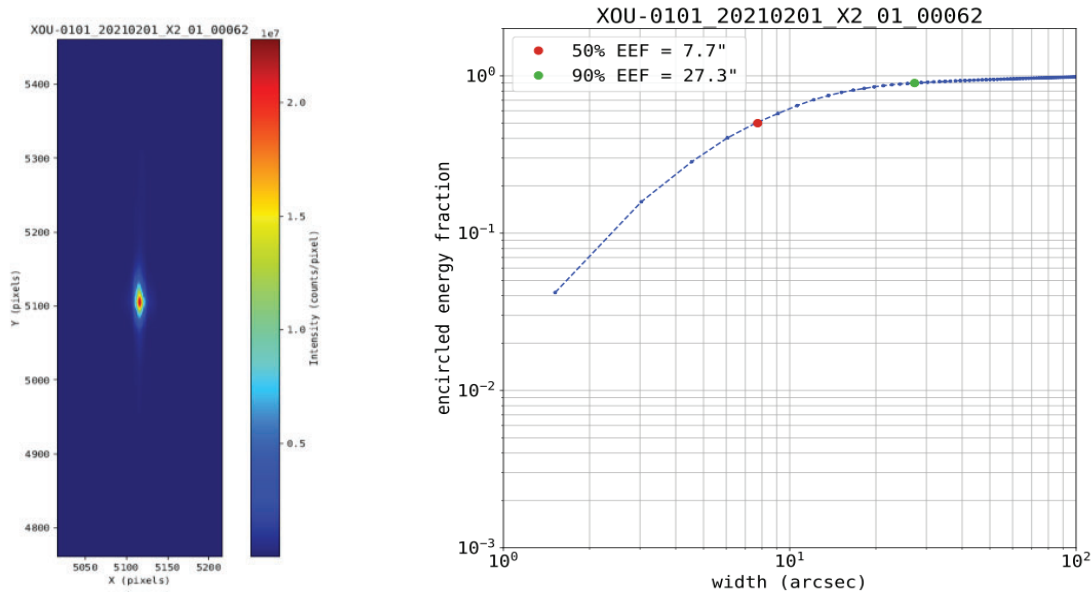

Figure 2: The PSF (left) and encircled energy fraction (right) for a middle radius XOU S/N 0101 , for a $70 \%$ wide contiguous area measured over 20 plates in double reflection at BESSY at $1 \mathrm{keV}$.

On outer radii we have, with an earlier generation stacking recipe which still lacks the SALEX improvements, also achieved a new bench mark, as shown in Figure 3.
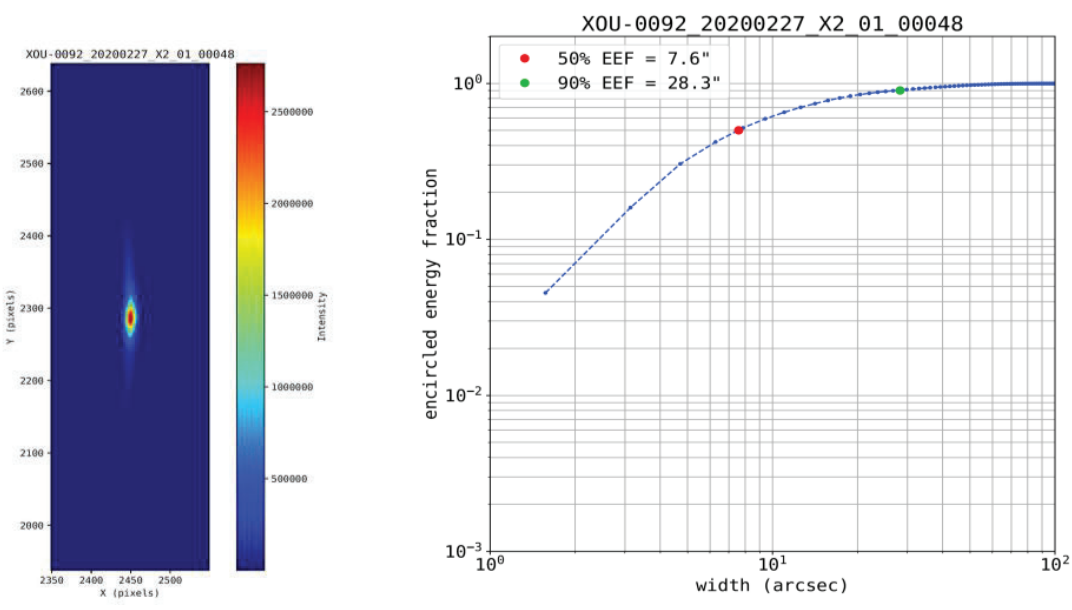

Figure 3: The PSF (left) and encircled energy fraction (right) for an outer radius XOU S/N 0092 , for a $70 \%$ wide contiguous area measured over 20 plates in double reflection at BESSY at $1.6 \mathrm{keV}$.

The lessons learned from the outer and middle radii stacking have also been applied to the inner radii stacking robot and in mid-2020 a performance of 16.9" was measured (see Figure 4) over 34 mirror pairs, a significant single step improvement compared to the earlier measured 60" performance.

It shall be noted that XOU-0101 is the very first attempt at making stacks that use the improved generation 6 stacking recipes. In the course of 2021 we will continue to implement many improvements on plate and stack level, as further discussed in the next chapters. The goal is to reach a 35 plate middle radius XOU performance significantly better than 10 " over $100 \%$ width and to push as large as possible an area below 7", as an important steps towards the Athena MAR. 

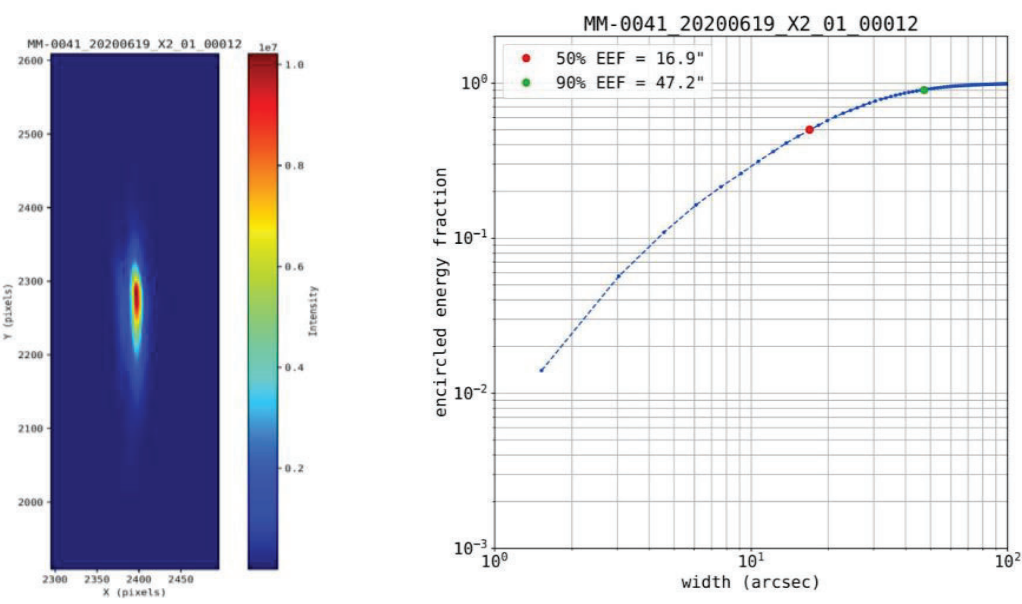

Figure 4: The PSF (left) and encircled energy fraction (right) for an inner radius XOU S/N 0094 , for a $70 \%$ wide contiguous area measured over 34 plates in double reflection at BESSY at $1.6 \mathrm{keV}$.

\section{MIRROR PLATE DEVELOPMENTS}

\section{Plate production}

As part of the preparations for the Athena MAR we continue upgrading the mass production capabilities [17] of our plate suppliers: Micronit Microfluidics and Teledyne e2v. A fully automated $300 \mathrm{~mm}$ wafer dicing system has been installed and commissioned at Teledyne imaging, which allows to increase the mirror plate production speed.

Most plates produced to date still contain a bias in their wedge (a 3.3" sloped layer etched into each mirror to make the optics focus in radial direction), which results in a stack-up error degrading the angular resolution. Dedicated metrology was initially developed by cosine to measure with $\mathrm{nm}$ accuracy the thickness of silicon and silicon oxide layers, which is present on both the ribbed and the reflective side of the mirror plates (see Figure 5). This led to the discovery of the bias, which is a result of having different etching rates on the reflective and ribbed side of the plates.
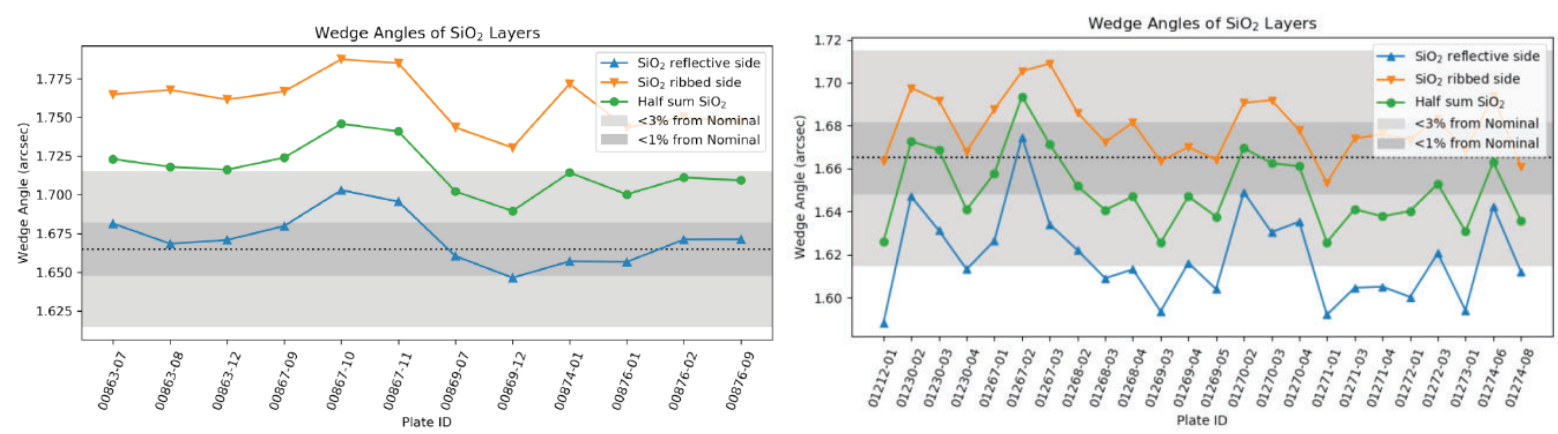

Figure 5: Wedge angle bias for a batch of 12 middle radius SPO plate (left). The green line is the half sum of the total oxide thickness and should be normally distributed around the design value (dashed line), but the oxide on the ribbed side of the plate is etched differently than on the reflective side and creates a bias, which results in a stack-up error. With dedicated metrology the bias can be reduced, and first batches of plates (right) show a reduced bias of $\mathbf{- 0 . 9 \%}$. The gray band indicate the allowed tolerance. We are currently working to achieve all production to be within a $1 \%$ from nominal band.

Micronit has recently received a copy of this dedicated metrology equipment to be able to measure the total thickness variation of the mirror plates and to adjust the etching process to remove the bias. With this equipment it will also be possible to further tighten the production tolerances, in order to produce plates that are within the target variation of less than $1 \%$ from the nominal value. 


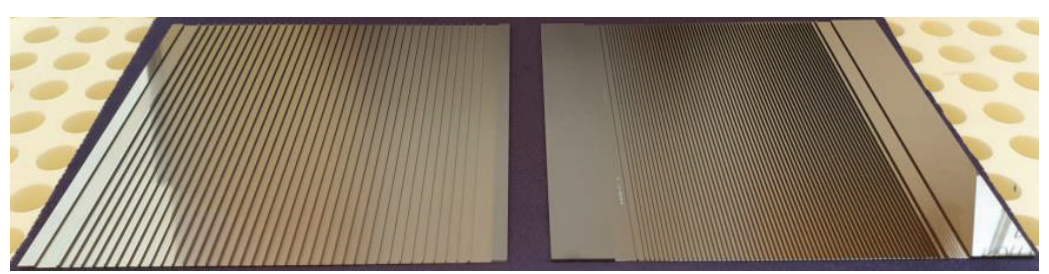

Figure 6: First prototypes of 'row 08' mirror plates (left). The increased rib spacing of $2.3 \mathrm{~mm}$ is clearly visible, when compared to existing $1 \mathrm{~mm}$ rib pitch plates (right).

Other activities concern the optimization of the plate shipment packaging (to reduce cost and increase cleanliness) and the development of new plate types that shall meet the target Athena optics design. An example is shown in Figure 6, which shows the first prototype 'row 08' (corresponding to the current middle radius plates used for process development) mirror plate, with an increased rib spacing of $2.3 \mathrm{~mm}$ and where we try to achieve the desired membrane thickness of $0.11 \mathrm{~mm}$, to maximize effective area and reduce off-axis vignetting. Larger quantities of such plates will only become available towards the second half of 2021 and will be used to transition the SPO development from the development radii to the 'real' Athena configuration.

\section{Mirror plate coating, lift-off and cleaning}

To enhance the $\mathrm{x}$-ray reflectivity high-z and low-z layers need to be sputtered onto the mirror plates. For the stacking process we require areas on the reflecting side of the plate, where the stiffening ribs make contact, to be coating free. This is achieved through a lithographic masking process with a subsequent lift-off after coating at cosine. ESA is currently procuring a dedicated spray coating machine for Micronit, to be installed in 2021. This system will allow the production of resist spray-coated plates with larger volume, throughput and consistent quality.
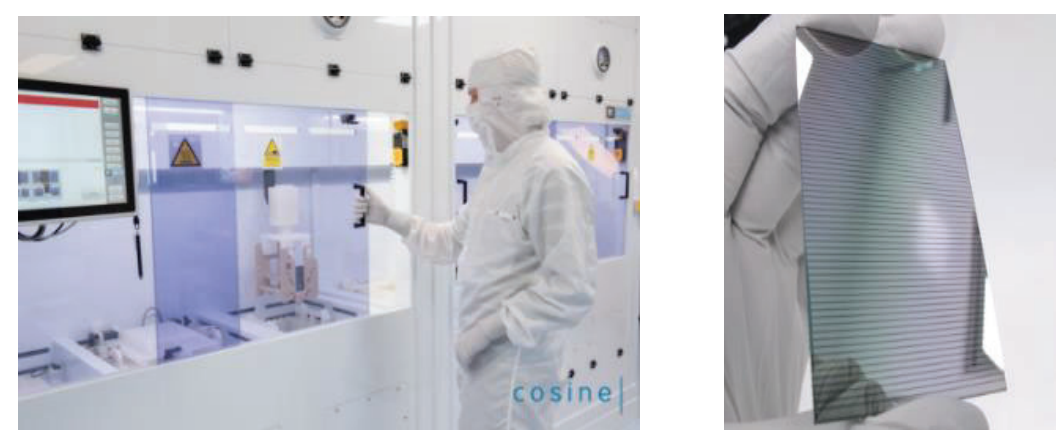

Figure 7: The newly installed fully automated cosine wetbench (left), which includes also an automated lift-off batch for post coating resist removal. The right photo shows a fully processed plate after lift-off, which was taken during the commissioning of the system in Nov 2020.

The mirror plate coating takes places at cosine using an already installed high-volume magnetron sputtering system [18], which also includes a plasma cleaning system [19]. The coating machine is currently being upgraded with a third magnetron to complete the system. It is then able to sputter iridium, chromium and either $\mathrm{SiC}$ or $\mathrm{B}_{4} \mathrm{C}$. A dedicated activity is running together with the Danish Technical University (DTU) to streamline the mass production process, with emphasis on first achieving a stable baseline for producing large quantities of clean and bondable iridium coated plates. This should be achieved in Q2/2021. We shall then proceed to add a low-z overcoating [20], again with emphasis on it surviving all process steps involved in making SPO plates.

The automated cleaning of SPO plates prior to stacking has also been further industrialised. A fully automated wetbench with handling robot has been installed and commissioned at cosine (see Figure 7): this new wetbench also includes an automated lift-off bath. The plates can be loaded in cassettes into the machine, and are automatically run through lift-off 
and SC-1 cleaning. The system includes chemical concentration monitors for quality assurance purposes and to achieve a reproducible cleaning process.

\section{STACKING PROCESS DEVELOPMENTS}

The stacking process development continues with high intensity. Four stacking robots (see Figure 8) are now available at cosine, which are each able to achieve a consistent 6.5 minutes per plate stacking time, already sufficient for the Athena production rates. The four robots have been harmonized to have similar interfaces to mount stacking equipment, such that production and development efforts can be run in parallel.

The stack quality is being further improved through further development of the stacking recipe, which depends not only on the type of die used to pre-shape the mirror plate prior to bonding, but also on the timing and sequence of releasing the plate. Significant resources are being used to develop finite element models of the stacking process. The models are used to study the influence of plate parameters (e.g. oxide thickness and stress, membrane thickness, rib spacing etc) and of stacking robot parameters on the stack quality in terms of meridional curvature, edge effects and stack-up error. One example is shown in Figure 9. Nevertheless, simulating an entire stack is still prohibitive in terms of computing time and we continue following the experimental route to improve the angular resolution step-by-step. The results, as shown in the HEW evolution Figure 1 show that optics quality keeps improving.

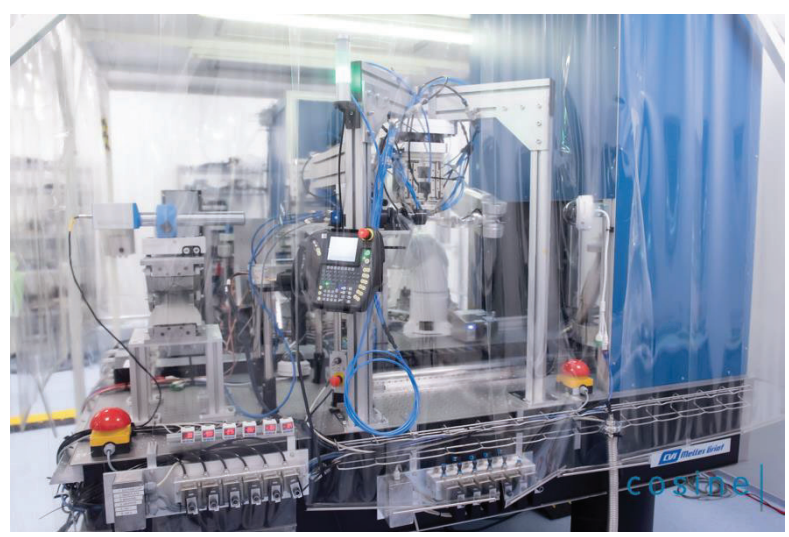

Figure 8: One of the four stacking robots, which is able to stack one plate within 6.5 minutes consistently. It is hosted on an optical table inside a cleanroom, is fully automated and includes in-line metrology that can characterize each mirror plate after having been stacked.
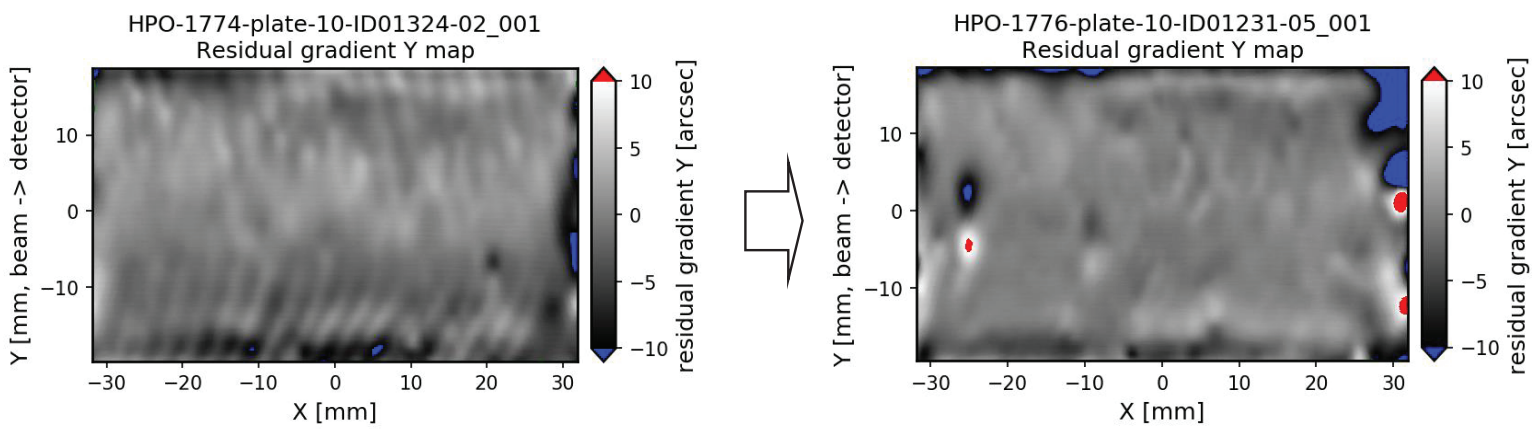

Figure 9: Example of the interaction between finite element modelling of the stacking process and improvements in practice. From the models a periodic structure was predicted to originate with a specific set of stacking parameters, which can be reduced by smoothening the trajectory of the hexapod. The stacking metrology data shows the before (left) and after (right) result of the process improvement. 
One important aspect of the stacking process development for the Athena MAR is the demonstration of a repeatable quality and of a repeatable production rate. To this end one of the four robots has been dedicated to pilot production, where dedicated laboratory technicians exercise the entire production cycle, from plate incoming inspection, over coating, lift-off, cleaning and stacking. The timing is recorded and analysed and process improvements are being implemented (see Figure 10).
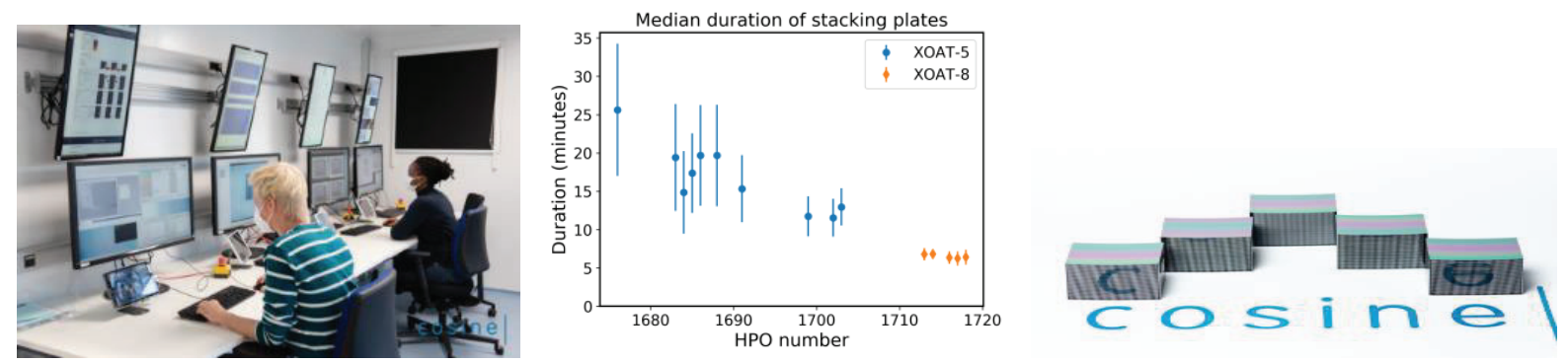

Figure 10: The stacking robots as shown in Figure 8 are remote controlled by laboratory technicians (left), who train producing stacks with repeatable quality in a reproducible timing. One of the earlier stacking robots (XOAT-5, middle) still included many manual steps, resulting in a variable stacking rate. XOAT-8, the newest stacking robot can stack plates with a cadence of $6.5 \pm 0.8$ minutes per plate. The resulting 5 stacks shown on the right, correspond to the 5 orange data points in the central figure.

\section{ENVIRONMENTAL TESTING}

Vibration, shock, acoustic and thermal cycling tests of the first 'row 08 ' modules are being prepared for 2021. In parallel we are now completing vibration and shock testing of inner, middle and outer radii mirror modules [21], as part of the technology development program. The Athena shock requirements are quite severe, as the optical bench will be mounted close to the lower end of the Ariane 6 fairing where it will experience significant shocks during the various phase of the launch [22]. Detailed finite element models of all rows of Athena have been produced to analyse their stiffness and the results flow into optimization of the so-called dowel pins that are glued to connect the mirror modules to the optical bench, but also to study the effects of the mirror plate design (thinner membrane, larger rib spacing) on the resonance frequencies and thermal behavior of the modules.
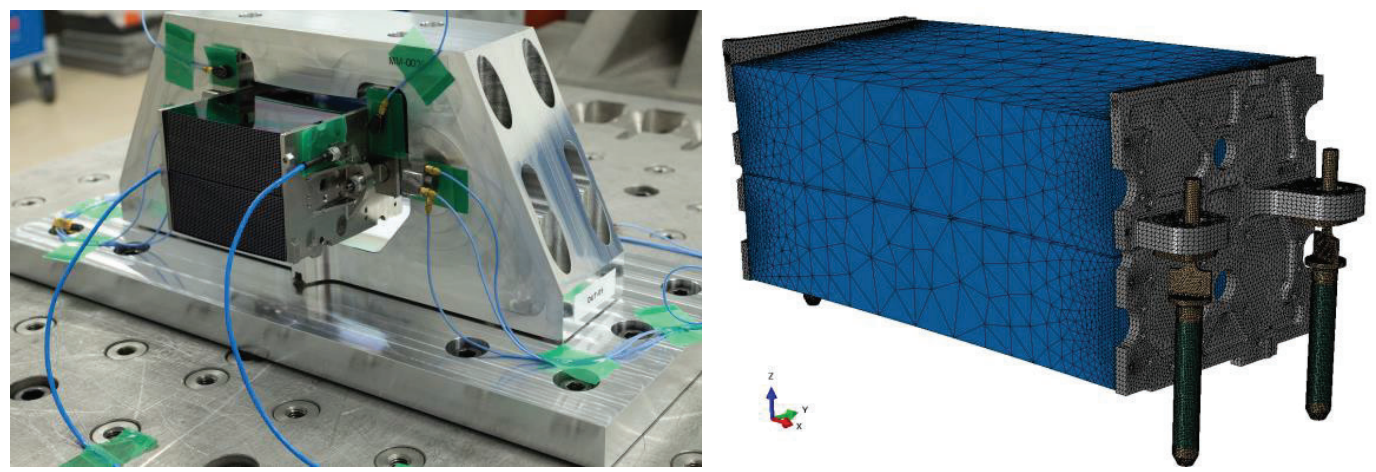

Figure 11: A middle radius mirror module during vibration testing (left). Detailed finite element model of a mirror module including the pins to mount the mirror module on the optical bench.

As mirror modules will have to undergo acceptance level qualification tests before being delivered for optical bench integration, we require test dowel-pins that can be connected to the mirror modules without using glue, for mounting during vibration and shock testing. These so-called 'dry mount' dowel pins are being development and will be used in the middle of 2021 for first testing with row-08 mirror modules. 


\section{CONCLUSION}

The Silicon Pore Optics development is progressing with high intensity towards the next Athena milestone, the Mission Adoption Review. All three evaluation criteria (angular resolution, effective area and mass production capabilities) are being worked on in parallel, using inner, middle and outer radii stacks and stacking robots. The angular resolution for all three radii has significantly improved during 2020. The mechanism that have deteriorated the stack quality towards the edges has been understood and solutions are being implemented, bringing $100 \%$ of middle radii XOUs below 10 " for the first time, with a good basis for further improvement towards the 5" optics goal. Mass production of plates, coating and stacks is being ramped up and first prototypes of coated mirror modules conform the latest Athena optics design are being prepared for $\mathrm{x}$-ray and environmental testing in 2021.

\section{ACKNOWLEDGMENTS}

We would like to acknowledge all of the excellent and hard work of the many people and institutions that contribute to the development of Silicon Pore Optics and especially the contribution of the laboratory technicians of cosine. Without their efforts, dedication and talent we would not be able to build the lens required for the largest x-ray observatory to be flown so far. We also like to acknowledge the ESA, NWO (Athena, 184.034.002) and MPE funding that makes the development of SPO possible.

\section{REFERENCES}

[1] K. Nandra, "Athena: Exploring the Hot and Energetic Universe", Proc. American Astronomical Society HEAD meeting (\#14), (2014).

[2] Meidinger, N., et al, "The Wide Field Imager instrument for Athena”, Proc. SPIE 9905, Space Telescopes and Instrumentation 2016: Ultraviolet to Gamma Ray, 99052A (18 July 2016)

[3] Barret, D., et al, "The ATHENA X-ray Integral Field Unit (X-IFU), Proc. SPIE 10699, Space Telescopes and Instrumentation 2018: Ultraviolet to Gamma Ray, 106991G (31 July 2018)

[4] Willingale, R. et al, "Science requirements and optimization of the silicon pore optics design for the ATHENA mirror", Proc. of SPIE Vol. 9144, 91442E (2014)

[5] Bavdaz, M. et al, "Optics developments for ATHENA", Proc. SPIE 11119, Optics for EUV, X-Ray, and Gamma-Ray Astronomy IX, 111190D (9 September 2019)

[6] Collon, M. et al, "Status of the silicon pore optics technology", Proc. SPIE 11119, Optics for EUV, X-Ray, and Gamma-Ray Astronomy IX, 111190L (12 September 2019)

[7] Wille, E., et al, "Silicon pore optics manufacturing plan and schedule for ATHENA", Proc. of SPIE Vol. 10699, 106993L (2018)

[8] Valsecchi, G., et al, "ATHENA Telescope: alignment and integration of SPO mirror modules", Proc. SPIE 11180, International Conference on Space Optics — ICSO 2018, 111801 N (12 July 2019)

[9] Ayre, M.. et al., “ATHENA: phase A study status and optics/instrument accommodation”, Proc. SPIE 11119, Optics for EUV, X-Ray, and Gamma-Ray Astronomy IX, 111190R (9 September 2019).

[10] Bavdaz, M., et al, “The ATHENA X-ray Optics Development and Accommodation”, same proceedings

[11] Vacanti, G, et al., "X-ray testing of silicon pore optics", Proc. SPIE 11119, Optics for EUV, X-Ray, and Gamma-Ray Astronomy IX, 111190I (9 September 2019).

[12] Krumrey, M., et al, "X-ray pencil beam facility for optics characterization”, Proc. SPIE 7732, 773240 (2010).

[13] Krumrey, M., et al, "New X-ray parallel beam facility XPBF 2.0 for the characterization of silicon pore optics", Proc. SPIE 9905 (2016).

[14] Burwitz, V. et al., "Developing, testing, and calibrating the ATHENA optics at PANTER", Proc. SPIE 10399, 1039900 (2017).

[15] Bradshaw, M., et al, "Developments in testing x-ray optics at MPE's PANTER facility", Proc. SPIE 11119, Optics for EUV, X-Ray, and Gamma-Ray Astronomy IX, 1111916 (9 September 2019)

[16] Keek, L., et al, "Stacking of mirrors for silicon pore optics", Proc. SPIE 11119, Optics for EUV, X-Ray, and Gamma-Ray Astronomy IX, $111190 \mathrm{H}$ (9 September 2019). 
[17] Landgraf, B., et al, "Development and manufacturing of SPO x-ray mirrors", Proc. SPIE 11119, Optics for EUV, X-Ray, and Gamma-Ray Astronomy IX, 111190E (30 October 2019).

[18] Massahi, S., et al, "Installation and commissioning of the silicon pore optics coatings facility for the Athena mission", Proc. SPIE 11119, Optics for EUV, X-Ray, and Gamma-Ray Astronomy IX, 111190F (9 September 2019).

[19] Girou, D., et al, "Plasma etching for the compatibility of thin film metallic coatings and direct bonding of silicon pore optics", Journal of Applied Physics. 128, 9, 7 p., 095302

[20] Svendsen, S., et al, "Performance and time stability of $\mathrm{Ir} / \mathrm{SiC}$ x-ray mirror coatings for ATHENA", Proc. SPIE 11119, Optics for EUV, X-Ray, and Gamma-Ray Astronomy IX, $111190 \mathrm{G}$ (9 September 2019).

[21] Barrière, N. M., et al, "Assembly of confocal silicon pore optic mirror modules", Proc. SPIE 11119, Optics for EUV, X-Ray, and Gamma-Ray Astronomy IX, 111190J (10 September 2019).

[22] Girou, D., et al, "Environmental testing of silicon pore optics for Athena", Proc. SPIE 11119, Optics for EUV, X-Ray, and Gamma-Ray Astronomy IX, 111190K (8 October 2019). 